\section{Temporal anagrams: Word identification with successively presented letters in scrambled order*}

\author{
IRWIN POLLACK † \\ University of Michigan, Ann Arbor, Michigan 41804
}

High-frequency seven-letter words were displayed letter by letter in scrambled temporal orders upon an oscilloscope. After each incorrect identification, the sequence was repeated. Highest word-identification scores were obtained with initial and terminal starting positions, with shortest and longest spacing between successive letters, and at the slowest and fastest rates of letter presentation. The relative contribution of each variable was maintained in combination with other variables. The conditional probability of a correct word identification, given that the word was not identified on a previous presentation, plunges sharply with successive presentations. This apparent nonindependence of performance over successive presentations is, however, shown to be an artifact of pooling the results of heterogeneous experimental variables.

Mayzner, Tresselt, and their associates have thoroughly examined two experimental situations ${ }^{1}$ : anagram problem solving (a review and bibliography is available in Tresselt, 1968) and perception of alphabetic sequences presented in irregular temporal orders (a review and bibliography is available in Mayzner et al, 1967). In the former experimental situation, a string of letters is presented, usually simultaneously, in an irregular spatial order. The task of the $S$ is to rearrange the letters to form a word. For example, "aichr" is presented. This anagram may be rearranged as "chair." In the latter experimental situation, a string of letters is presented sequentially in an irregular temporal order, but in the correct spatial order. For example, "chair" may be presented in successive frames as: $* \mathrm{~h} * * *$, $\mathrm{c}^{* * * *}, * *_{\mathrm{a}} * *, * * * * \mathrm{I}, * * *_{\mathrm{i}} *$, where $*$ signifies a blank in the respective sequence position.

At extremely rapid rates of irregular sequential presentation, individual elements may be "blanked out" under a specified range of spatial-temporal constraints. For example, in the example cited above, the " $h$ " can be blanked out if the "c" and "a" are plotted within about $100 \mathrm{msec}$ of the "h." Unlike the case of anagrams, there is little spatial uncertainty, but there is uncertainty with respect to the identity of the individual letters at each location.

At slower rates of presentation, the individual letters are each perceived. With

*This research was aided in part by National Science Foundation Grant GB 14036X. Thanks are due to Mrs. Kathryn Weidner who supervised the experiments, to Louis Wojnarowski who wrote the computer program, and to Robert Hsieh for processing the experimental data.

+Mental Health Research Institute, The University of Michigan, Ann Arbor, Michigan 48104. imperfect memory for spatial position, however, the task resembles the solving of an anagram in that spatial uncertainty replaces letter uncertainty. For this reason, the term "temporal anagram" appears to be appropriate to the perception of letters presented within slow irregular temporal sequences. On the scale of "perceptual to cognitive," such displays are more clearly associated with the cognitive integration of scrambled parts than in the perceptual recognition of individual characters.

With temporal anagrams, we might expect better performance with more rapid rates of presentation, except at rates at which sequential blanking is obtained. Rapid sequential presentation unburdens memory for spatial location. In the extreme case, successive letters are presented so rapidly that, irrespective of the order of plotting, the entire sequence appears to be plotted simultaneously. If memory for sequential position were the sole limitation upon the solution of temporal anagrams, we might expect poorer performance at slower and slower rates of presentation. However, at extrenely slow rates of presentation, control operations (cf. Atkinson \& Schiffrin, 1968), such as rehearsal, measurement of spatial location relative to some boundary markers, etc., could be employed. Poorest performance might therefore be obtained at intermediate rates of presentation.

The aim of the present tests was to examine factors in the solution of temporal anagrams obtained by extending the Mayzner-Tresselt paradigm of irregular letter presentation to slower rates of presentation. A secondary din was to test whether or not the responses to successive presentations of temporal anagrams can be regarded as independent.

\section{METHOD}

Materials and Experimental Design

Seven-letter words were selected from the Thorndike-Lorge word list. Words were first selected from the list of most common words, and successive selections were made at decreasing word frequencies until 343 words were selected. The same 343 words were employed in two differently scrambled sets. Each set consisted of three subsets of 112,112 , and 119 words each. One-half of the 24 Ss completed each set. Within each set, the order of presentation of each subset of conditions was approximately counterbalanced, and the starting position within each subset was randomized. In a 7 by 7 by 7 design of three variables, each condition of each variable occurred within each block of 7 trials, and each 7 by 7 two-way combination of two variables at a time occurred within each block of 49 trials.

\section{Display Conditions}

Individual words were presented letter by letter upon a Tektronics 602 display scope equipped with a P4 (green) phosphor. The display was driven by a PDP.9 computer. The preexposure, interexposure, and postexposure fields were relatively dark. The background level at the display was about $0.01 \mathrm{~mL}$; the level of the displayed letters was about $4 \mathrm{~mL}$ as measured by an S.E.I. photometer, Letters were constructed by 5 by 7 dot arrays. The height of each letter array was $.75 \mathrm{~cm}$, the width was $.5 \mathrm{~cm}$, and the width of the entire seven-letter word was $7.5 \mathrm{~cm}$. (All distances are for fine dots; typical focus adjustments yielded dot diameters of about $0.5 \mathrm{~mm}$.) The viewing distance was about $65 \mathrm{~cm}$.

The following display variables were manipulated: the time of presentation between successive letters, the starting letter, and the spatial distance between successively presented letters. There were seven conditions of each variable: interletter durations of I to 64 temporal units of $1 / 60 \mathrm{sec}$, each of the seven possible starting positions, and each of the possible six spatial distances plus a random scrambling among letters. The on time of each letter was 1 unit of $1 / 60 \mathrm{sec}$. Two specific examples are presented in Fig. 1 for the word BETWEEN, with an on time of each letter at 1 time unit and an interstimulus interval (ISI) between letters at 2 time units. The left side of Fig. 1 illustrates a starting position (SP) of 1 and a distance (D) between successive letters of 1. The right side of Fig. 1 illustrates a starting position of 4 and a distance between successive letters of 2 . In each case, a still camera open for 19 time units would record BETWEEN upon a single 


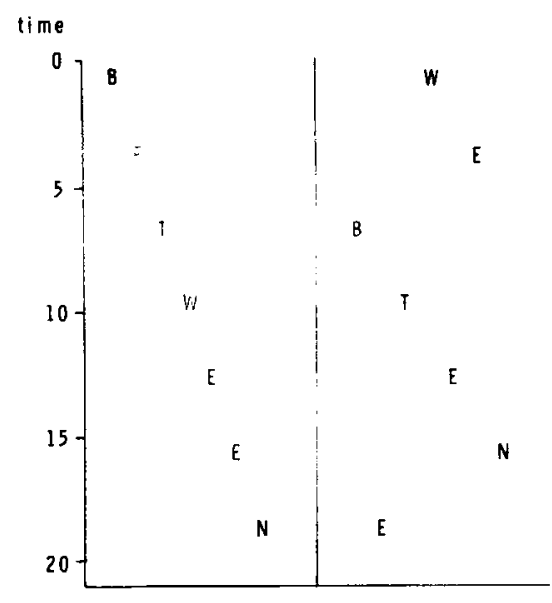

spatial position $\rightarrow$

Fig. 1. Illustration of two experimental conditions in the sequential plotting of successive letters of the word BETWEEN.

horizontal line. To the extent that the eye does not fully integrate the display over time, different temporal orders may be perceived. Note that the spatial position of each letter within the word is independent of its temporal order of presentation.

\section{Subjects}

Twenty-four university students, experienced in auditory psychoacoustical testing, served as Ss. Each S served under each of the 343 conditions for a total of 8,232 trials.

\section{Procedure}

A trial was initiated by the $S$ by pressing a key on the teletypewriter. The $S$ than typed in his best guess, with knowledge that the vocabulary consisted only of seven-letter common English words. The S was also permitted to indicate that he could not make a reasunable guess. A correct response terminated the trial. The same word was repeated in the same temporal-spatial order following each incorrect response until seven successive incorrect responses were obtained. The correct answer was furnished after the seventh incorrect response.

\section{RESULTS}

Overall Difficulty Level of the Tests

The overall level of word identification was high. Table 1 lists the number of trials which required $1,2, \ldots 7$, and $>7$ successive representations. Of 8,232 trials (24 Ss by 343 experimental conditions), 5,409 , or $65.7 \%$, required only a single presentation. Also presented in Table 1 is the conditional probability of a correct response at each successive presentation. given that the response was incorrect upon the previous presentation. The conditional probability of a correct response falls rapidly with successive presentations. About $5.7 \%$ of the trials were terminated after seven incorrect responses.

An interesting measurement problem arises. Since a majority of the trials required only a single presentation, medians are not an appropriate measure. The crude, noninterpolated median number of successive presentations was one for 270 of 343 conditions, was two for 50 conditions, was three for 14 conditions, was four for 4 conditions, and was five or more for only 2 conditions. The mean is also inappropriate for truncated distributions. A linear extension of the cumulative frequency distribution for the results in Table 1 intersects $100 \%$ at about Trial 14, suggesting that the center of the truncated portion is in the vicinity of 11 presentations. A curvilinear extension results in an even higher estimate. Nevertheless, all calculations of means will assume that all trials not completed after seven successive presentations were completed upon the eighth presentation. The method is conservative with respect to differences among experimental conditions.
Table 1

Number of Correct Responses on Successive Presentations

\begin{tabular}{ccc}
\hline Presentations & $\begin{array}{c}\text { Correct } \\
\text { Responses }\end{array}$ & $\begin{array}{c}\text { Conditional } \\
\text { Probability }\end{array}$ \\
\hline 1 & 5409 & .657 \\
2 & 1337 & $.474^{\mathrm{b}}$ \\
3 & 506 & .341 \\
4 & 235 & .240 \\
5 & 125 & .168 \\
6 & 83 & .135 \\
7 & 67 & .125 \\
\hline 7 & $470^{\mathrm{a}}$ & \\
\hline
\end{tabular}

aTrials, out of 8232 , remaining after 7 incorrect responses.

b 1337/(8232-5409)

\section{Experimental Variables}

Figure 2 presents three performance measures associated with each of the variables examined one at a time (and collapsed over the other two). The lop row is the proportion of initial responses which were correct, the symbols representing individual conditions which yield the best, the worst, and median performance; the second row is the proportion of trials not correct after seven successive presentations relative to the total number of trials, and, as dashed lines, relative to the number of

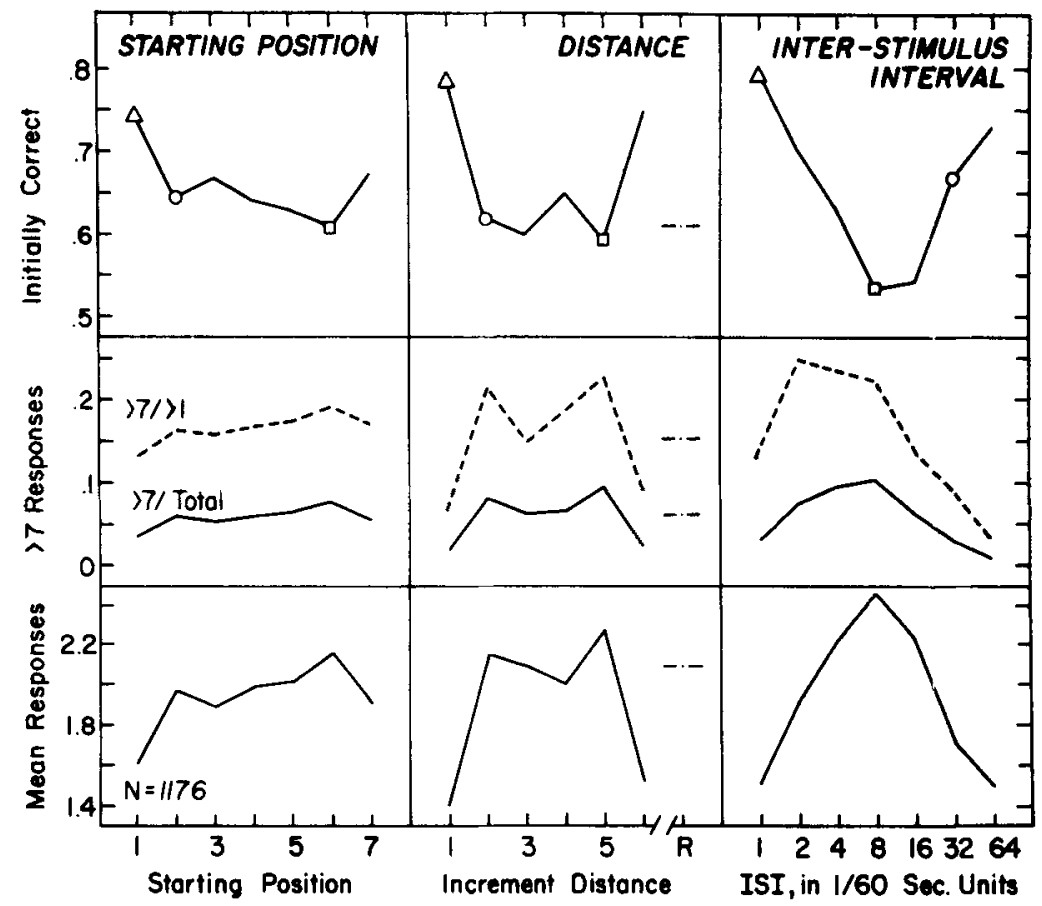

Fig. 2. Summary results for each of the individual conditions of each of three variables. The top row represents the proportion of trials in which the word was correctly identified on the first presentation. The symbols demark the condition with the highest, the lowest, and the median number of correct initial trials. The middle row represents the probability of $>7$ incorrect responses; the dashed curve represents the conditional probability of $>7$ incorrect responses, given that the first response was incorrect. The bottom row represents the mean number of responses, assuming a correct response on the eighth presentation after 7 incorrect responses. 
Table 2

Best-Worst Analysis of Pairs of $V$ ariables

\begin{tabular}{|c|c|c|c|c|c|c|}
\hline & \multicolumn{3}{|c|}{ Best } & \multicolumn{3}{|c|}{ Worst } \\
\hline & $\mathrm{SP}$ & $\mathbf{D}$ & ISI & $\mathbf{S P}$ & $\mathbf{D}$ & ISI \\
\hline \multicolumn{7}{|c|}{ Starting Positions (SP) } \\
\hline 1 & & 1 & 1,64 & & $3, \mathrm{R}$ & 16 \\
\hline 2 & & 1 & $2,1,64$ & & 5 & 8 \\
\hline 3 & & 6,1 & 1,64 & & 2 & 8 \\
\hline 4 & & 6 & 1 & & 5,3 & 16 \\
\hline 5 & & 6,1 & 1 & & 5,3 & 8,16 \\
\hline 6 & & 1 & 1,64 & & 5 & 16 \\
\hline 7 & & 6,1 & 1 & & $\mathbf{3}, \mathbf{R}$ & 8.16 \\
\hline \multicolumn{7}{|c|}{ Distance (D) } \\
\hline 1 & 1 & & $1,64,2$ & 4 & & 8 \\
\hline 2 & 1 & & 1,64 & 6 & & 8 \\
\hline 3 & 1 & & 1 & 5 & & 8 \\
\hline 4 & 1,6 & & 1 & 2,5 & & 16 \\
\hline 5 & 1 & & 1,64 & 6.2 & & 16 \\
\hline 6 & 7,1 & & 64,1 & 6 & & 16 \\
\hline $\mathbf{R}$ & 1,5 & & 1 & 2 & & 16 \\
\hline \multicolumn{7}{|c|}{ Interstimulus Interval (ISI) } \\
\hline 1 & 1 & 6.1 & & 6 & 2 & \\
\hline 2 & 1,7 & 6.1 & & 3 & $\overline{5}$ & \\
\hline 4 & 7,1 & 1,6 & & 6 & $2,5, \mathrm{R}$ & \\
\hline 8 & 1 & 6,1 & & $\mathbf{5}$ & 3 & \\
\hline 16 & 2,1 & 1 & & 5,4 & $5,3, R$ & \\
\hline 32 & 1 & 1.6 & & 6 & 5 & \\
\hline 64 & 1 & 6,1 & & 5,2 & 3 & \\
\hline
\end{tabular}

trials remaining after an initial incorrect response; the bottom row is the mean number of successive presentations for a correct response, assuming eight presentations for those trials not completed after seven incorrect responses. It is noted that, with the exception of the conditional measure of the middle row, the several measures are necessarily correlated with each other. The left column of Fig. 2 considers the effect of the starting position; the middle column examines the effect of incremental distances; and the right column examines the effect of interstimulus interval.

Best performance is achieved at the first and at the last starting positions, at the shortest and at the largest incremental distances, and at the fastest and slowest rates of presentation. (Note that an initial starting position of 1 with an incremental distance of 1 "spells" out the word in the forward order, whereas a starting position of 7 and a distance of 6 spells out the word in a backward order.) Poorest performance is associated with intermediate conditions of the separate variables. The poor performance associated with intermediate conditions did not result from the order of testing of conditions. The order was scrambled within and across Ss.

Analysis of variance of the initially correct responses revealed that each of the three variables, their two-way interactions, and their three-way interactions were significant at the 0.001 level, when evaluated against the corresponding mean-square term including Ss. In addition, Ss and the interaction with distance and
SI were significant at the 0.001 level when evaluated against the four-way interaction as well as three-way interactions including $S s$ were not significant. The four-way interaction of $\mathrm{Ss}$ and the three variables accounted for only $0.5 \%$ of the total factorial variance.

A "best and worst" analysis of pairs of conditions is presented in Table 2. For each entry in the left column, the value of the "other" variable which gave rise to the best and worst average scores is given as entries. For example, for a fixed starting position of 1 , the largest number of correct responses on the initial trial was obtained with Distance 1 (data collapsed over ISI), and with ISI of 1 and 64 (data collapsed over distance). When two conditions of a given variable were about equally effective, they are separated by a comma.

The best-worst pairwise analysis also tends to reflect the first-order analysis: best scores with SP at 1 and 7,D at 1 and 6 , and ISI at 1 and 64 ; poorest scores at SP of 5 and $6, D$ of 3 and 5, and ISI at 8 and 16 time units. One interaction concerns the regularity of presentation $(D=1, S P=1$; $\mathrm{d}=6, \mathrm{SP}=7$ ).

The combination of pairs of variables is illustrated in Fig. 3 in terms of the proportion of initially correct responses. The six sections show the combination of the two variables represented by the abscissa and parameter, with scores averaged over the third variable. For example, the upper left corner considers scores under seven starting positions (abscissa) for those three conditions of distance (parameter) which yielded the highest, the lowest, and the median scores. The main point of Fig. 3 is that there are not extremely sharp interactions among the variables: the curves representing the highest and lowest conditions of one variable do not intersect.

A best-worst triplet analysis (over the 24

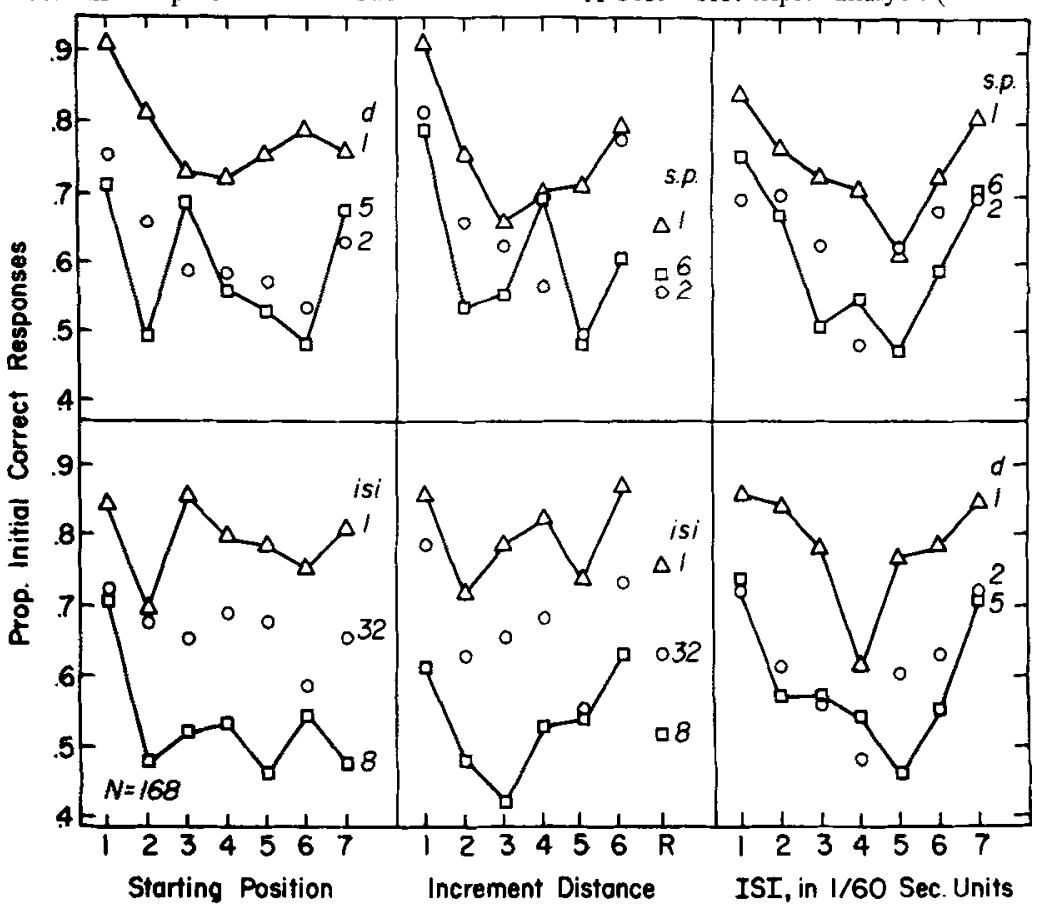

Fig. 3. Proportion of trials with a correct initial response for combinations of conditions represented by the abscissa and the parameter. 


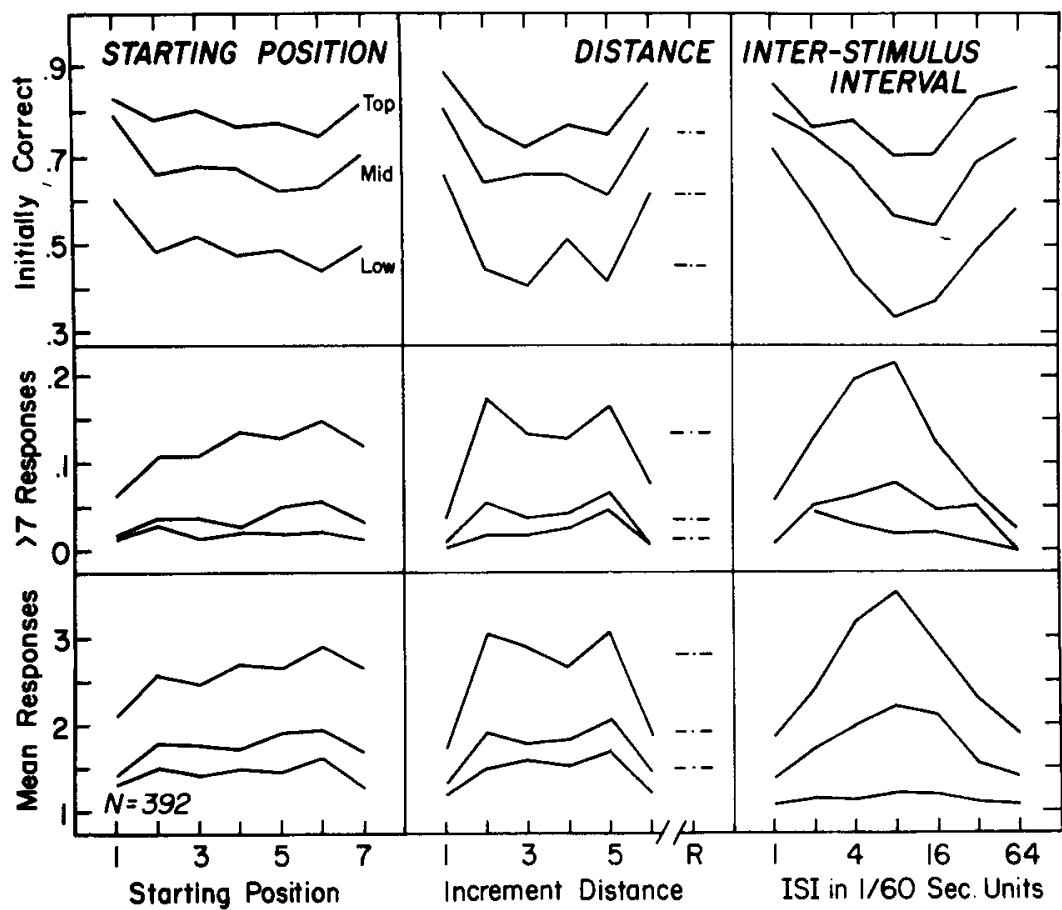

Fig. 4. Summary results for individual conditions associated with three subgroups of Ss differing in overall proficiency. As in Fig. 2.

Ss) also tended to verify the stability of rank of the individual conditions within each variable.

\section{Individual Differences}

In light of Mayzner et al's (1969) finding that some Ss respond differentially at fast and at slow rates, a search was made for sharp interactions with overall $S$ proficiency.

With respect to independence upon successive presentations, the conditional probability of a correct response was higher on Presentation I than on Presentation 2 for each of the $24 \mathrm{Ss}$; higher for 23 Ss on Presentation 2 than on Presentation 3; higher for 19 Ss on Presentation 3 than on Presentation 4; higher for 17 Ss on Presentation 4 than on Presentation 5; and higher for 18 Ss on Presentation 5 than on Presentation 6. Clearly, the observed nonindependence of Table 1 does not result from $S$ pooling.

The 24 Ss were divided into three groups based upon their overall number of correct initial responses. The analyses represented in Fig. 4 were carried out for the individual subgroups. No substantial changes are noted in terms of the gross ordering of the variables. The range of scores, with respect to the individual conditions, is greatest with the poorest subgroup and is least with the best subgroups of Ss. These differences may more nearly reflect upon the specific measures employed and possible "ceiling effects" than upon real differences among the subgroups. anagrams of the present study. Since a general model for the solution of anagrams has been proposed by Tresselt (1968), this discussion will be confined to the following question.

\section{Are Successive Presentations Independently Identified?}

The results of Table 1 argue strongly for nonindependence for the present situation in which the individual items are accurately perceived. Figure 5 presents a conditional probability analysis of the accuracy of word identification on the initial presentation or on successive trials, given that the previous response was incorrect. The left panel considers the results averaged over 49 combinations of distance (D) and interstimulus interval (ISI) for the starting position (SP) with the highest correct identification on the first presentation (triangles), for the starting position with the lowest incorrect identification on the first presentation (squares), and for the median condition (unconnected circles). The middle and right panels are similarly organized as a function of the selected conditions of distance and interstimulus interval.

The major point of Fig. 5 and of Table 1

There have been several recent interesting reports on the perception of letters "overprinted" upon the same display area (Haber \& Nathanson, 1969; Kolers \& Katzman, 1966; Mayzner et al, 1970; Newman, 1966; Troxel, 1967). These studies appear to be more concerned with the perception of individual units than with the scrambled temporal may become discouraged following an

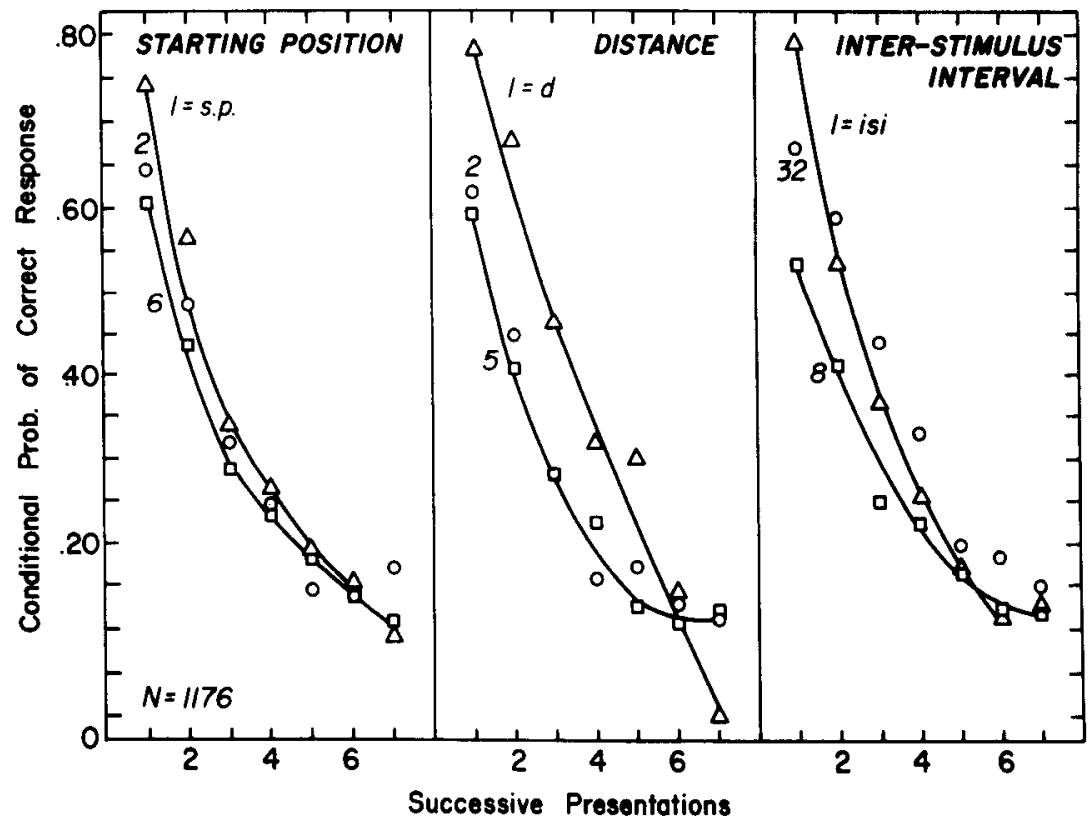

Fig. 5. Conditional probability of a correct response upon successive presentations for selected experimental conditions. 
incorrect response, especially when they know that the to-be-identified word will be presented for a fixed number of incorrect trials. A partial way out of the problem is to follow Haber (1969) and employ a variable number of successive presentations. This procedure was not employed because such a large proportion of trials were correct on the first presentation.

A second reason for the nonindependence is that heterogeneous sources are contributing to the average score, and that the apparent nonindependence is simply a statistical artifact of averaging. For example, assume two Ss of vastly different skill levels. Each $S$ demonstrates independence upon successive presentations, but one identifies 0.9 of the remaining words on each presentation, and the other identifies 0.1 of the remaining words. With 1,000 presented words, the first $S$ identifies 900 , 90 , and 9 words on the first three presentations; the second $S$ identifies 100 , 90 , and 81 words on the first three presentations. The average over the two Ss is 500,90 , and 45 words for a conditional probability of correct identification of $0.50,0.18$, and 0.11 , respectively. Had the second $\mathrm{S}$, instead, identified 0.8 of the words on each presentation, his scores would have been 800,160 , and 32 , resulting in average scores of 850,125 , and 20.5 for a conditional probability of 0.85 , 0.83 , and 0.82 , respectively. In general, the wider the heterogeneity, the further will be the apparent departure from independence, even when independence holds for the individual factors. Confining the results to a single condition (and pooling over other variables), therefore, does not necessarily produce a constant conditional probability of correct response.

In order to avoid pooling the effects of different experimental variables, the individual 343 experimental conditions were analyzed. With only 24 responses available per condition, sufficient data were available only to examine the change in conditional probability of a correct response between the first and second presentations. The conditional probability decreased for 210 conditions, increased for
128 conditions, and was the same for 2 conditions. Perfect scores were obtained on the initial trials for 3 conditions. Thus, even when reduced to the individual experimental condition (with pooling over $24 \mathrm{Ss}$, half exposed to one word and half to another), nonindependence remains.

The third reason for nonindependence is intimately related to the second and may differ only with respect to emphasis. Instead of viewing nonindependence as a statistical artifact introduced by averaging over nonhomogeneous conditions, we might view nonindependence as an outcome of experimental design. In the present tests, two words were assigned to each experimental condition. Had the two words differed sharply in difficulty, a source of nonhomogeneity would have been introduced. It is ironic that counterbalancing procedures which distribute word difficulty more evenly also tend to negate the opportunity to make some important substantive tests. A conditional probability analysis was performed for each of the two words under each of the 343 conditions. A total of 646 words were not received perfectly (12 Ss out of 12) on the first presentation. The conditional probability of a correct response decreased from the first to second presentation for 319 words, increased for 311 words, and was the same for 16 words. Thus, the effects of individual variables have been sufficiently titrated to arrest completely the sharp plunge of conditional correct response with successive presentations of Table 1 and Fig. 5.

Might other demonstrations of nonindependence across successive presentations also yield independence under such an analysis? And what implication is to be drawn when independence across successive presentations is demonstrated, even when pooling takes place over both words and Ss (Haber, 1967)?

In summary, for the grand aggregate across all conditions, within each condition of each experimental variable or within a single experimental condition, nonindependence of word identification was found on successive presentations. Only with isolation of separate words within single experimental conditions does one obtain independence upon successive presentations.

\section{REFERENCES}

ATKINSON, R. C., \& SCHIFFRIN, R. M. Human memory: A proposed system and its control processes. In K. W. Spence and J. T. Spence (Eds.), Advances in the psychology of learning and motivation research and theory. Vol. II. New York: Academic Press, 1968. Pp. 89-195.

HABER, R. N. Repetition as a determinant of perceptual recognition processes. In $W$. Wathen-Dunn (Ed.), Models for the perception of speech and visual form. Cambridge: M.I.T. Press, 1967. Pp. 202-210. [Also in R. N. Haber (Ed.), Information processing approaches to visual perception. New York: Holt, Rinehart \& Winston, 1969. Pp. 273-279.]

HABER, R. N., \& NATHANSON, L. S. Processing of sequentially presented letters. Perception \& Psychophysics, 1969, 5, 359-361.

KOLERS, P. A., \& KATZMAN, M. T. Naming sequentially presented letters and words. Language \& Speech, 1966, 9, 84-95.

MAYZNER, M. S., TRESSELT, M. E., \& HELFER, M. S. A provisional model of visual information processing with sequential inputs. Psychonomic Monograph Supplements, 1967. 2(7, Whole No. 23),

MAYZNER, M. S., TRESSELT, M. E., TABENKIN, N., DIDNER, R., \& HELFER, $M$. $S$. Visual information processing of sequentially presented inputs: I. Effects of input timing on sub-span storage and retrieval mechanisms. Perception \& Psychophysics, $1969,5,297-302$.

MAYZNER, M. S., TRESSELT, M. E., CHECKES, J., \& HOENIG, H. A. Visual information processing of sequentially presented inputs: III. Further effects of list length and interstimulus interval values on sub-span storage and retrieval mechanisms. Perception \& Psychophysics, 1970, 7, 294-296.

NEWMAN, E. B. Speed of reading when the span of letters is restricted. American Journal of Psychology, 1964, 272-278.

TRESSELT, M. E. A reexamination of anagram problem solving. Transactions of New York Academy of Sciences, 1968, 30, 1112-1119.

TROXEL, D. E. Experiments in tactile and visual reading. IREE Transactions on Human Factors in Electronics, 1967, HFE-8. 261-263.

NOTE

1. This paper is dedicated to the memory of M.E. Tresselt who contributed so actively to both experimental situations.

(Accepted for publication October 1, 1970.) 